\title{
Fast Quenching the Burst of Host Salicylic Acid Is Common in Early Strawberry/Colletotrichum fructicola Interaction
}

\author{
Chengyong He,1,2 Ke Duan,1,2,† Liqing Zhang, ${ }^{1}$ Ling Zhang, ${ }^{1,3}$ Lili Song, ${ }^{1,2}$ Jing Yang, ${ }^{1}$ Xiaohua Zou, ${ }^{1}$ \\ Yanxiu Wang, ${ }^{3}$ and Qinghua Gao ${ }^{1, \dagger}$
}

\begin{abstract}
${ }^{1}$ Shanghai Key Laboratory of Protected Horticultural Technology, Forestry and Fruit Tree Research Institute, Shanghai Academy of Agricultural Sciences (SAAS), Shanghai 201403, China; ${ }^{2}$ College of Food Science and Technology, Shanghai Ocean University, Shanghai 201306, China; and ${ }^{3}$ College of Horticultural Sciences, Gansu Agricultural University, Lanzhou 730070, China
\end{abstract}

Accepted for publication 12 August 2018.

\begin{abstract}
The fungus Colletotrichum fructicola (a species of C. gloeosporioides complex) causes devastating anthracnose in strawberry. Like other species of the genus Colletotrichum, it uses a composite strategy including both the biotrophic and necrotrophic processes for pathogenesis. Host-derived hormones are central regulators of immunity, among which salicylic acid (SA) is the core defense one against biotrophic and hemibiotrophic pathogens. However, the manner and timing of pathogen manipulation of SA are largely elusive in strawberry. To achieve better understanding of the early challenges that SA-mediated defense experiences during strawberry/C. fructicola interaction, dynamic changes of SA levels were followed through the high-performance liquid chromatography method. A very early burst of free SA at $1 \mathrm{~h}$ postinoculation (hpi) followed by a fast quenching during the next $12 \mathrm{~h}$ was noticed, although rhythm variations were present in two hosts. Transcriptional characterization of genes

were all induced at different time points. At the same time, three types of genes encoding homologous effectors interfering with SA accumulation were found to be first inhibited but sequentially activated during the first 24 hpi. Furthermore, subcellular localization analysis suggests that CfShyl is a weapon of $C$. fructicola for strawberry invasion. Based on these results, we propose that the infection strategy that $C$. fructicola utilizes on strawberry is specialized, which is implemented through the optimized expression of a specific set of effector genes. Transcriptional characterization of host genes supports that de novo SA biosynthesis and the free SA release from methyl salicylate might contribute equally to the intricate control of SA homeostasis in strawberry. C. fructicola manipulation of SA-dependent resistance in strawberry might be closely related to multihormonal interplay among SA, jasmonic acid, abscisic acid, and cytokinin.
\end{abstract} related to SA pathway in two varieties on $C$. fructicola inoculation revealed that these genes were differentially expressed, although they
Additional keyword: mycology.
The genus Colletotrichum is a large fungal genus, and most of its members are important plant pathogens. In this genus, Colletotrichum gloeosporioides species complex is the notable one associated with $>470$ different host species as the major causal agents of many important commercial fruit crops, such as avocado, olive, orange, banana, mango, coffee, and strawberry (Hyde et al. 2009). In C. gloeosporioides species complex, the genome of $C$. fructicola subsp. Nara gc5 (Weir et al. 2012), an estimated total size of which is $55.6 \mathrm{Mb}$, was isolated and sequenced (Gan et al. 2013).

Strawberry (Fragaria $\times$ ananassa Duch.) is one kind of worldwide favorite fruit crop; it is also vulnerable to C. gloeosporioides, which can cause anthracnose and crown rot, resulting in great yield losses. Until now, these diseases developed without a successful control by chemicals (Baroncelli et al. 2015; Buddie et al. 1999; DenoyesRothan et al. 2003; Freeman and Katan 1997; Garrido et al. 2008; Han et al. 2016; Jelev et al. 2008; Rahman et al. 2015). C. gloeosporioides uses a hemibiotrophic infection strategy to colonize the host strawberry, and nearly all cultivated varieties are susceptible to

†Corresponding authors: K. Duan; E-mail: kduan936@126.com, and Q. Gao; E-mail: qhgao20338@sina.com

Funding: This work was supported by funds from National Natural Science Foundation of China grants 31501592 and 31601731 and Science and Technology Commission of Shanghai Municipality Key Basic Research Project grant 14JC1405400 and Key Program grant 16391901400.

*The $\boldsymbol{e}$-Xtra logo stands for "electronic extra" and indicates that five supplementary tables and two supplementary figures are published online.

The author(s) declare no conflict of interest.

(c) 2019 The American Phytopathological Society these fungi to varying degrees (Amil-Ruiz et al. 2011). Notably, C. gloeosporioides-caused anthracnose has brought significant losses in recent years in middle and eastern China, mainly in the middle lower regions of the Yangtze River where warm, moist climate and abundant rainfall favor the epidemic spread (Han et al. 2016; Ren et al. 2008; Xie et al. 2010; Zhang et al. 2016). However, the invasion strategies of $C$. gloeosporioides and the interplay between strawberry and the pathogens are largely unknown.

Plant hormones are crucial for plant growth and survival, and the modulation of plant immunity is actually a matter of hormones (Pieterse et al. 2012). Multiple hormones are the central players triggering plant immunity, and their signaling pathways are interconnected in a complex network, whereas pathogens try to manipulate host hormone-regulated signaling (Asai and Shirasu 2015). The balance of hormone pathways determines resistance to particular pathogen (Vos et al. 2013). The small phenolic compound salicylic acid (SA) is a multifaceted hormone for plants to combat disease, which is especially crucial for the resistance to biotrophic and hemibiotrophic pathogens (Gimenez-Ibanez and Solano 2013). It is involved in multiple resistance-related processes consisting of basal resistance and effector-triggered immunity (ETI) as well as systemic acquired resistance (SAR) (Lu et al. 2016). At the same time, biotrophic and hemibiotrophic pathogens have evolved efficient strategies to down-regulate SA signaling via targeting SA biosynthesis and signaling (Patkar and Naqvi 2017; Tanaka et al. 2015).

SA-mediated defense signaling and the extent to which it is manipulated by pathogen are elusive in strawberry. Several studies have suggested the involvement of SA in the strawberry/ Colletotrichum pathosystem. Ectopic expression of nonexpressor of pathogenesis-related genes 1 (AtNPR1) increased strawberry resistance to $C$. gloeosporioides (Silva et al. 2015). Microarray 
analysis integrated with the quantitative polymerase chain reaction (qPCR) method identified the partial activation of an SA-mediated defense pathway in strawberry on $C$. acutatum infection, and enrichment of transcripts of the SA pathway was detected after 1 day postinoculation (dpi) (Amil-Ruiz et al. 2016). Temporal accumulation of SA at $48 \mathrm{~h}$ postinoculation (hpi) was observed in strawberry plants challenged by the avirulent strain of $C$. fragariae but not by the virulent strain of $C$. acutatum (Grellet-Bournonville et al. 2012). Heat shock at $50^{\circ} \mathrm{C}$ for $20 \mathrm{~s}$ increased free SA accumulation in strawberry leaves 2 days after treatment, which may be responsible for the resistance enhancement of the strawberry plants to $C$. gloeosporioides inoculated 1 day after heat shock (Widiastuti et al. 2013). In a recent work, we reported a transient increase of free SA in the less susceptible variety Sweet Charlie instead of the susceptible one Jiuxiang at $6 \mathrm{hpi}$, and external SA pretreatment elevated the internal SA levels in both varieties followed by a reduced susceptibility (Zhang et al. 2016). The early stage of plant-pathogen interaction is crucial for the successful invasion and pathogenesis. To reveal the SA-related defense mechanism and multihormonal signaling network underlying strawberry responses to anthracnose fungus, we endeavor to comprehensively characterize the SA homeostasis and signaling at the biochemical and transcriptional levels in this pathosystem during the first $24 \mathrm{hpi}$.
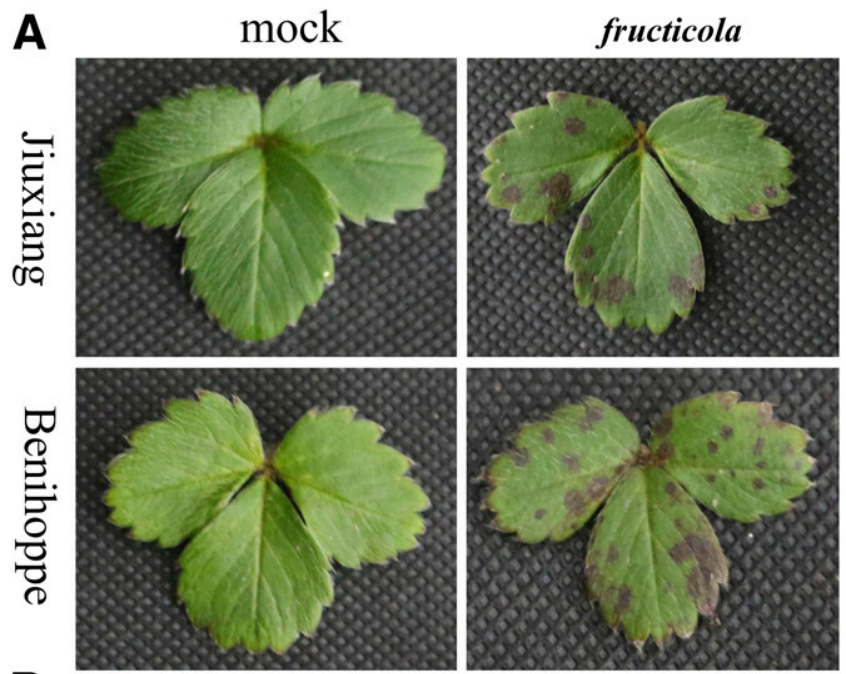

B

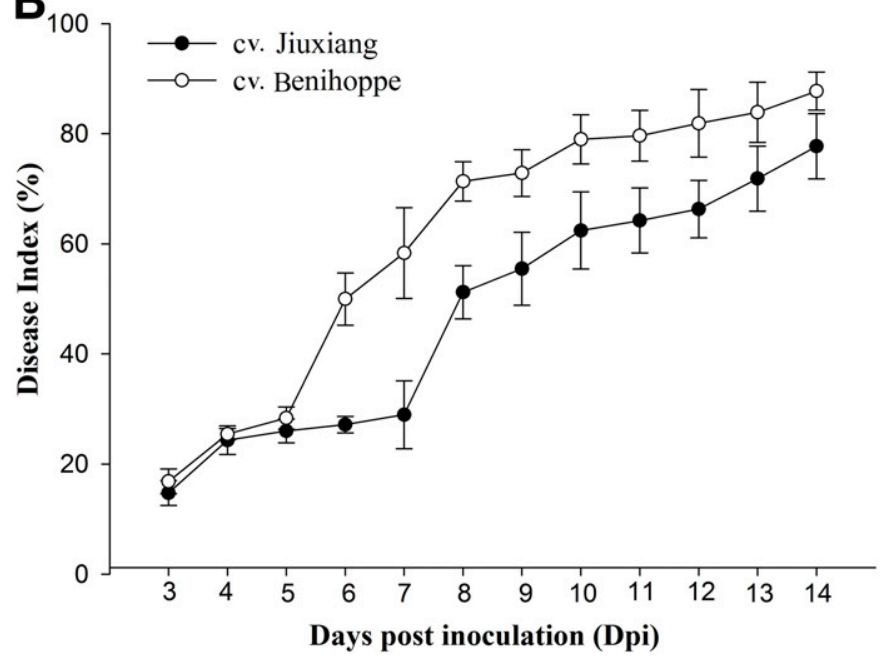

Fig. 1. The susceptibility of strawberry cultivars Jiuxiang and Benihoppe to Colletotrichum fructicola. A, Anthracnose symptom on leaves at 9 days postinoculation (dpi). B, Disease index on whole strawberry plants from 3 to 14 dpi with $C$. fructicola. Disease index was based on a zero to five rating scale applied to all leaves on each plant (Zhang et al. 2016). Data are shown as mean \pm standard deviation ( $n=3$ biological replicates).
In this research, SA accumulation and expression profiles of related genes were characterized during the first $24 \mathrm{~h}$ in two susceptible strawberry varieties infected by $C$. fructicola. By using the high-performance liquid chromatography (HPLC) method, the levels of SA in strawberry leaves after C. fructicola inoculation were determined. Dynamic analysis revealed a noticeable transient increase followed by a fast quenching of this burst during the first $24 \mathrm{~h}$ of infection. Quantitative reverse transcription-polymerase chain reaction (PCR) was performed to uncover the transcriptional responses of the related genes, including those among the SArelated defense system and hormone marker genes in strawberry, as well as three gene families in C. fructicola targeting SA homeostasis. Our work sheds light on the hormone signaling network at an early phase of invasion, especially the manipulation of the SA-related pathway by $C$. fructicola, which regulates host defense and susceptibility to anthracnose.

\section{MATERIALS AND METHODS}

Plant materials and cultivation. Two strawberry varieties were used in this study, namely Jiuxiang and Benihoppe. Seedlings developed from the apical meristems of stolons through micropropagation were transplanted to the hole tray with moss peat (Pindstrup Substrate) and grown in a greenhouse for 6 months. Next, healthy plants with 8 to 10 compound leaves were transplanted into pots and cultivated in a growth chamber (Conviron, Adaptis A1000AR) for 1 month before inoculation. Conditions in the growth chamber were set as follows: $12 \mathrm{~h}$ light/day, $125 \mu \mathrm{mol}$ $\mathrm{m}^{-2} \mathrm{~s}^{-1}$ photo flux density, $70 \%$ relative humidity, and constant $25^{\circ} \mathrm{C}$.

Fungal pathotype and inoculum preparation. The $C$. fructicola isolate CGMCC3.17371 of C. gloeosporioides species complex used in this study was first collected in 1996 from diseased strawberry in Qingpu District, Shanghai (Ren et al. 2008). This isolate was maintained on a potato/dextrose/agar medium in the dark at $28^{\circ} \mathrm{C}$, and it was rejuvenated by infecting strawberry and reisolating single-spore culture every 2 years in our laboratory since 2008. Multilocus phylogenetic analysis assigned this isolate to $C$. fructicola species (unpublished data). For inoculum preparation, conidia were washed from 10- to 12-day-old culture with distilled water and adjusted to the desired concentration with a hemacytometer.

Inoculation and sampling. Plant inoculation was done as previously described (Zhang et al. 2016). In each experiment, 36 plants divided into three replicates were sprayed with conidial suspensions adjusted to a concentration of $10^{6 / 1} \mathrm{ml}$ with $0.01 \%$ (vol/ vol) Tween-20. Simultaneously, 36 additional plants were mock treated with sterile $\mathrm{diH} 2 \mathrm{O}$ containing $0.01 \%$ (vol/vol) Tween-20. The third to sixth fully expanded compound leaves from eight plants in each replicate were randomly harvested at $0,1,2,6,12$, and 24 hpi. The leaves from eight plants were pooled as a repetition and independently repeated three times. For 0 hpi, sampling was accomplished immediately after treatment (within $5 \mathrm{~min}$ ). Leaf blades were sampled and immediately frozen in liquid nitrogen; then, they were transferred to $\mathrm{a}-74^{\circ} \mathrm{C}$ freezer. For RNA isolation and SA measurement, the same (infected or mock-treated) plant powder homogenized by liquid nitrogen was utilized in 1 week after sampling.

Development of anthracnose symptom was scored on the other four plants in each replicate at 4, 7, and 14 dpi. Quantification of disease index was performed as previously described (Zhang et al. 2016) and independently repeated twice in different seasons. Similar results were obtained, and the result of one experiment was shown.

Selection of SA-related genes in the genomes of strawberry and $\boldsymbol{C}$. fructicola. Annotated strawberry genes related to SA synthesis, metabolism, binding, and signaling were obtained by keywords combined with Arabidopsis homologs basic local alignment search tool (BLAST) search of the strawberry 
genome (https://www.rosaceae.org/species/fragaria/fragaria_vesca /genome_v1.0). The molecular features of obtained protein sequences were further confirmed by Pfam server (http://pfam.xfam. org/search/sequence) with default settings against only Pfam-A families $(E$ value $=1.0)$. Previously reported pathogen genes targeting host SA biosynthesis and signaling were used to search against $C$. fructicola subsp. Nara gc5 isolate genome (https://www. ncbi.nlm.nih.gov/assembly/GCA_000319635.1). Keyword search and Pfam analysis further validated the identity of these $C$. fructicola genes. The lists of corresponding genes in strawberry and C. fructicola genomes are shown in Supplementary Tables S1 and S2, respectively.

RNA isolation and transcript analysis. Total RNA was extracted using an EASYspin RNA isolation kit with minor modification. The quality and quantity of RNA were determined as previously described (Li et al. 2013), and cDNA was synthesized using the PrimeScript RT Reagent Kit with gDNA Eraser according to the manufacturer's instructions. For reverse transcription, $1 \mu \mathrm{g}$ of RNA was used. The cDNA samples were subjected to real-time qPCR using SYBR Green Premix Ex Taq in accordance with the manufacturer's instructions. qPCR was performed on a thermocycler of Light Cycler 480 with the following settings: 2 min at $95^{\circ} \mathrm{C}$ followed by 40 cycles of $15 \mathrm{~s}$ at $95^{\circ} \mathrm{C}, 30 \mathrm{~s}$ at $58^{\circ} \mathrm{C}$, and $30 \mathrm{~s}$ at $72^{\circ} \mathrm{C}$. Data were analyzed using the Roche Light Cycler 480 II software. For $C$. fructicola, $\alpha$-tubulin (CGGC5_11160) and actin (CGGC5_6011) genes were used as references. For strawberry, FaEF1 $\alpha$ (gene28639) was used as the reference gene (Amil-Ruiz et al. 2013). We used the method of $2^{-\Delta \Delta \mathrm{Ct}}$ to calculate the relative expressions of strawberry and fungal genes, and the value of the control group at 0 hpi was set as one. Three technical replicates and three biological repeats were conducted for each sample. Primer sequences used are listed in Supplementary Tables S3 and S4.

Quantification of SA and glucosylated SA contents. Free SA and one of the common SA derivatives, glucosylated salicylic acid (SAG), in strawberry leaf blades were extracted and measured by HPLC following the protocol as previously described (Zheng et al. 2015) with minor modifications. In brief, SA was extracted from $\sim 100 \mathrm{mg}$ of leaf tissues first by methanol followed by ethyl acetate-cyclopentane (1:1 in volume). To convert SAG into SA, $20 \mu \mathrm{l}$ of $12 \mathrm{~N} \mathrm{HCl}$ was added to the organic phase after original SA extraction, incubated at $80^{\circ} \mathrm{C}$ for $60 \mathrm{~min}$, and then, centrifuged at maximum speed for $5 \mathrm{~min}$. The final extracts for SA and SAG were vacuum dried followed by nitrogen gas blowing. The dried extracts were then dissolved into HPLC eluent $(10 \%$ methanol in $0.2 \mathrm{M}$ acetate buffer) in a final volume of $300 \mu \mathrm{l}$ for free SA and $1,800 \mu \mathrm{l}$ for SAG-converted SA. The HPLC system consisted of a Waters
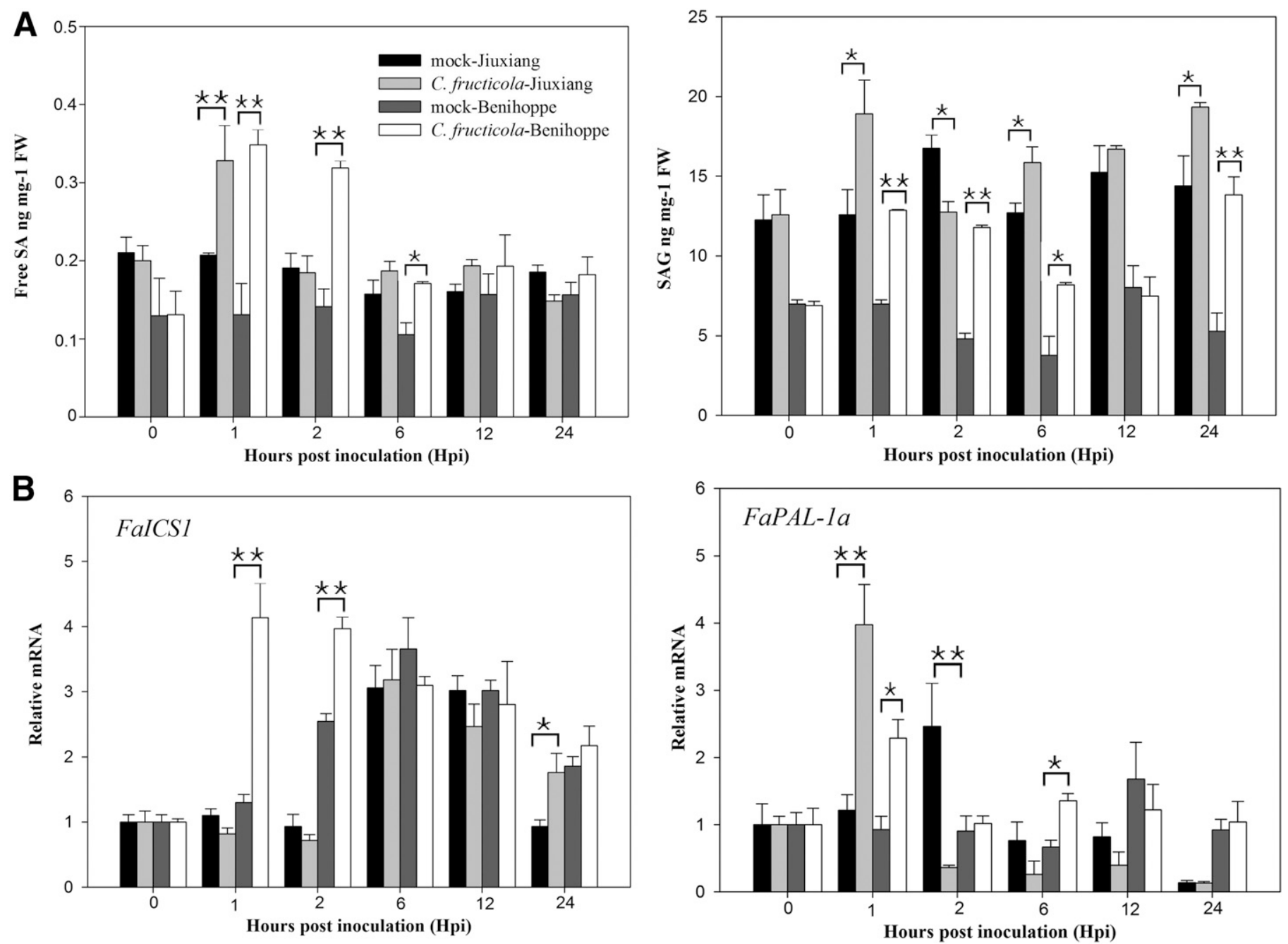

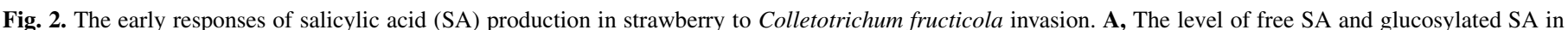

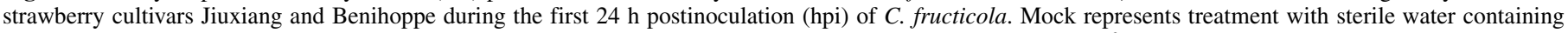

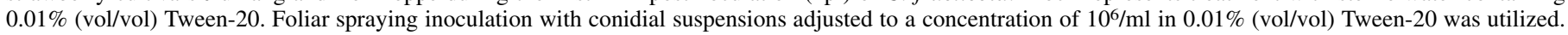

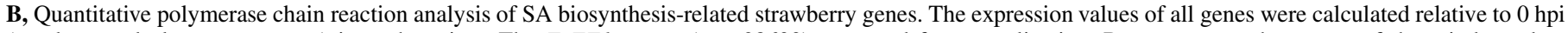

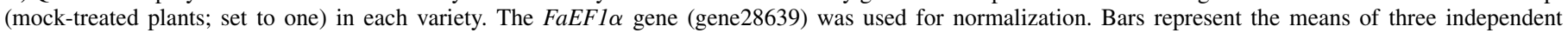

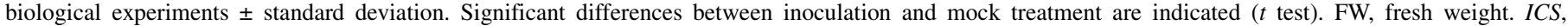
isochorismate synthase; $P A L$, phenylalanine ammonia lyase. ${ }^{*} P$ value $<0.05$; $* * P$ value $<0.01$. 
E2695 automatic injector and pump, an XTerraRMS C18 main column $(3.0 \times 100 \mathrm{~mm}, 3.5 \mu \mathrm{m})$ protected by an XTerraRMS C18 guard column $(3.0 \times 20 \mathrm{~mm}, 3.5 \mu \mathrm{m})$ placed in a column oven set at $25^{\circ} \mathrm{C}$, and a fluorescence detector (FLD-2475; Waters) with excitation wavelength at 295 to $305 \mathrm{~nm}$ and emission wavelength at 405 to $407 \mathrm{~nm}$. The total run time for each sample was $30.5 \mathrm{~min}$ at a flow rate of $0.3 \mathrm{ml} / \mathrm{min}$. SA peak came out at around $13.5 \mathrm{~min}$.

Vector construction and subcellular localization analysis. To generate the effector-green fluorescent protein (GFP) fusion construct, a fragment covering CfShyl open reading frame and its native promoter region was amplified by PCR. The PCR product was cotransformed into Saccharomyces cerevisiae strain XK125 together with XhoI-linearized pYF11 as previously described (Huang et al. 2017). The resulting yeast plasmid pYF11-CfShyl was expressed in Escherichia coli and confirmed by sequencing before being transformed into $C$. fructicola protoplasts. The original pYF11 plasmid was introduced into $C$. fructicola as a control. PCR primers used are listed in Supplementary Table S5.

The strawberry seedlings of Benihoppe with four to five leaves developed from micropropagation were inoculated with the fluorescent $C$. fructicola strain harboring pYF11 or pYF11-CfShyl. The growth conditions for the inoculated seedlings were same as previously described. Photographs were taken at 24 hpi under Zeiss LSM880 confocal laser scanning microscopy.

Statistics. For pairwise comparisons of SA/SAG contents between the pathogen-inoculated and mock-treated samples, Student's $t$ tests were performed in Microsoft Excel 2007. For multiple comparisons of data, a two-way analysis of variance (Statistical Analysis System software) was performed to determine the significance.

\section{RESULTS}

The development of $C$. fructicola-caused anthracnose symptoms in two strawberry varieties. Symptoms on leaves caused by $C$. fructicola in both Jiuxiang and Benihoppe were first observed at 3 dpi. Typical symptoms on a single compound leaf at 9 dpi are shown in Figure 1A. The anthracnose lesions similarly developed in two varieties during the first 3 to 5 dpi. Rapid development of typical necrosis occurred after 5 dpi in Benihoppe and after 7 dpi in Jiuxiang. Comparatively, Benihoppe harbored more lesions than Jiuxiang on leaves at 9 dpi, whereas Jiuxiang displayed a lighter symptom from 3 to 14 dpi (Fig. 1B). The two varieties were susceptible to $C$. fructicola, and their pathogenesis similarly developed at both the invasion and early necrotrophic stages (necrosis symptom/lesion noticeable). However, Jiuxiang suffered from a lighter disease than Benihoppe at the latter necrotrophic stage (after 6 dpi).

Dynamic changes of SAs in two varieties during the first 24 hpi. Under the same controlled conditions, basal levels of free SA in Jiuxiang and Benihoppe (the third to sixth fully expanded leaves; 10:00 a.m.) were 0.20 and $0.16 \mathrm{ng} \mathrm{mg}^{-1}$ fresh weight (FW), respectively (Fig. 2A). On $C$. fructicola infection, the levels of free $\mathrm{SA}$ in both varieties quickly changed. Compared with the mock treated, free SA levels in C. fructicola-infected Jiuxiang and Benihoppe rose by 80 and $126 \%$ at $1 \mathrm{hpi}$, reaching the maxima ( 0.36 and $0.38 \mathrm{ng} \mathrm{mg}^{-1} \mathrm{FW}$, respectively) during the first $24 \mathrm{~h}$. However, this burst of free SA was soon quenched in Jiuxiang at about 2 hpi. Comparatively, in Benihoppe, the pathogen-incited increase of free SA amount lasted longer (over $6 \mathrm{~h}$ ).

The accumulation of SAG, the main conjugated SA, also changed in the first day after inoculation. Consistently, SAG levels increased significantly after $C$. fructicola infection at $1 \mathrm{hpi}$ in both two varieties. This infection-triggered increase of SAG continued to be observed at 6 and $24 \mathrm{hpi}$. The dynamic changes in free SA and SAG were largely similar in Benihoppe, except that the level of free SA stayed constant after $12 \mathrm{hpi}$, whereas the SAG level rose again at 24 hpi. In Jiuxiang, however, the levels of free SA and SAG changed differently after 1 hpi.

In plants, SA may be produced from chorismate via either the isochorismate synthase (ICS)- or phenylalanine ammonia lyase (PAL)-mediated steps. The transcript level of strawberry ICS1 homolog (gene25950) significantly increased at 1 and 2 hpi in Benihoppe (Fig. 2B). A moderate rise was also observed at $24 \mathrm{hpi}$ in Jiuxiang for FaICS1 transcripts. The expression of FaPAL-1a homolog (gene23261) was induced at $1 \mathrm{hpi}$ in two varieties, which went down soon at 2 hpi in Jiuxiang but rose sharply at 6 hpi in Benihoppe. These results suggested that both ICS- and PALmediated pathways may be involved in SA biosynthesis in strawberry on $C$. fructicola infection.

Transcriptional responses of potential methyl salicylate esterase genes after $\boldsymbol{C}$. fructicola challenge. Methyl salicylate (MeSA) is a conserved long-distance SAR in the genus Arabidopsis and tobacco. Salicylic acid-binding protein 2 (SABP2) is an MeSA esterase, which is required to release the active defense SA from
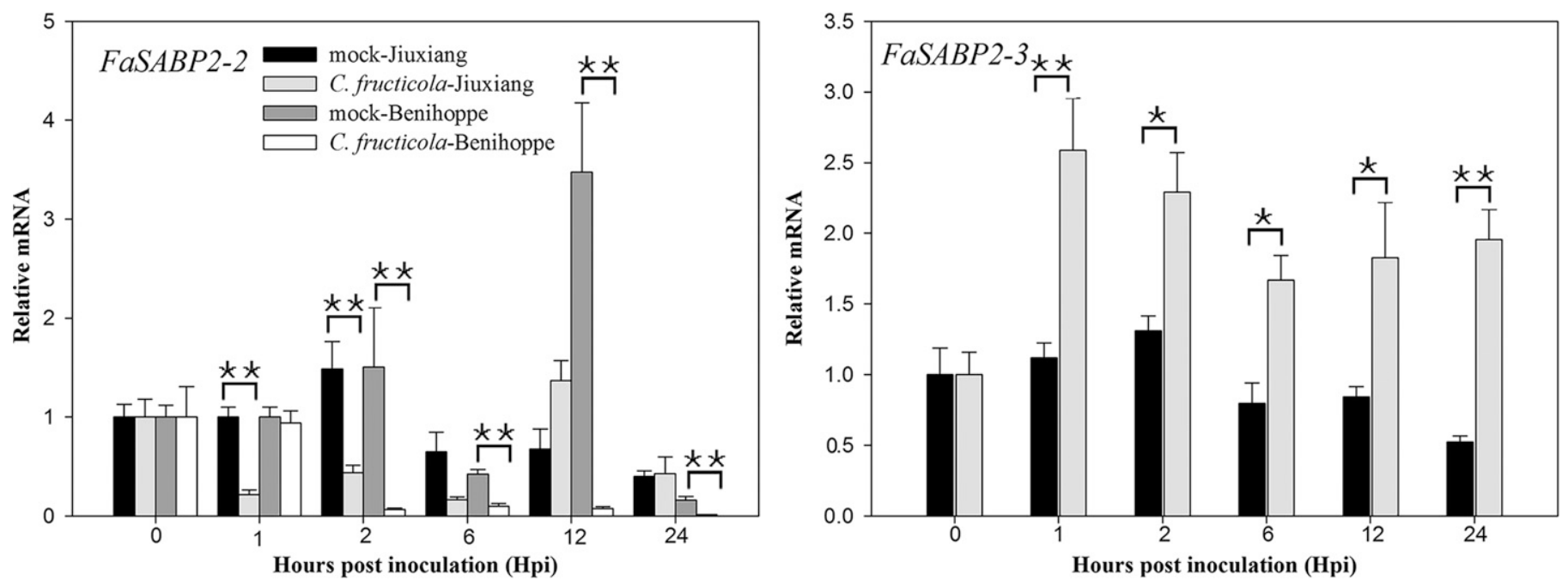

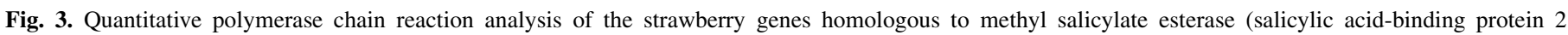

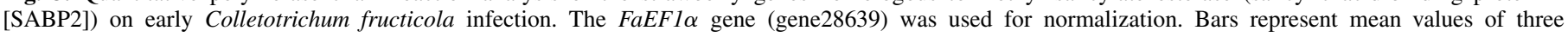

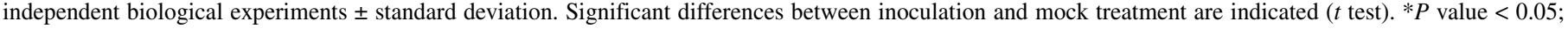
$* * P$ value $<0.01$. 
MeSA (Vlot et al. 2008). Strawberry reference genome harbors six SABP2 homolog genes. Phylogenetic analysis sorts these members into three subgroups (Supplementary Fig. S1).

Four members of potential SABP2-like genes were detected in the current $C$. fructicola/strawberry pathosystems (SABP2-2, -3, -5, and -6 ). qPCR analysis showed that transcription levels of SABP2-5 and SABP2-6 were both too low to be repeatedly detected during the first 24 hpi. The dynamic expression patterns of SABP2-2 (gene29393) and SABP2-3 (gene29424) are present in Figure 3. $S A B P 2-2$ transcription displayed significant decreases in two varieties with differential patterns. In Jiuxiang, $S A B P 2-2$ was suppressed at 1,2, and 6 hpi but mildly up-regulated at 12 hpi. By contrast, in Benihoppe, this gene expression was significantly and stably impaired in the first $24 \mathrm{hpi}$. The transcription level of $S A B P 2-$ 3 was stably up-regulated in the infected Jiuxiang, whereas it was not detected in Benihoppe. Clearly, the genes encoding SABP2 were moderately activated at a certain time point (12 hpi for $F a S A B P 2-2)$ or during the first 24 hpi (FaSABP2-3) in Jiuxiang, whereas no similar transcriptional activation was detected in
Benihoppe. These observations suggest that free SA release from MeSA may be activated in Jiuxiang but not in Benihoppe on C. fructicola infection.

Dynamic changes of $C$. fructicola genes encoding potential effectors targeting host $\mathrm{SA}$ production during the early invasion. To invade plant tissues, fungal pathogens have efficient strategies to down-regulate host SA biosynthesis. The chorismate mutase $(\mathrm{Cmu})$ converts $\mathrm{SA}$ precursor chorismate to prephenate, thus lowering host SA levels (Djamei et al. 2011). The salicylate hydroxylase (Shy) can directly degrade SA for a carbon source required by the pathogen (Rabe et al. 2013). The isochorismatase (Isc) rechannels isochorismate, the direct precursor of SA, into 2,3-dihydro-2,3-dihydroxybenzoate and pyruvate (Liu et al. 2014). All three types of genes encoding homologous effectors interfering with SA accumulation were identified in the C. fructicola genome, including 2 loci for Cmu, 13 loci for Shy, and 10 loci for Isc (Supplementary Fig. S2).

Transcripts of a total of eight pathogen genes (one $\mathrm{Cmu}$, four Shy, and three Isc loci) were detected in this pathosystem. Of the three Isc
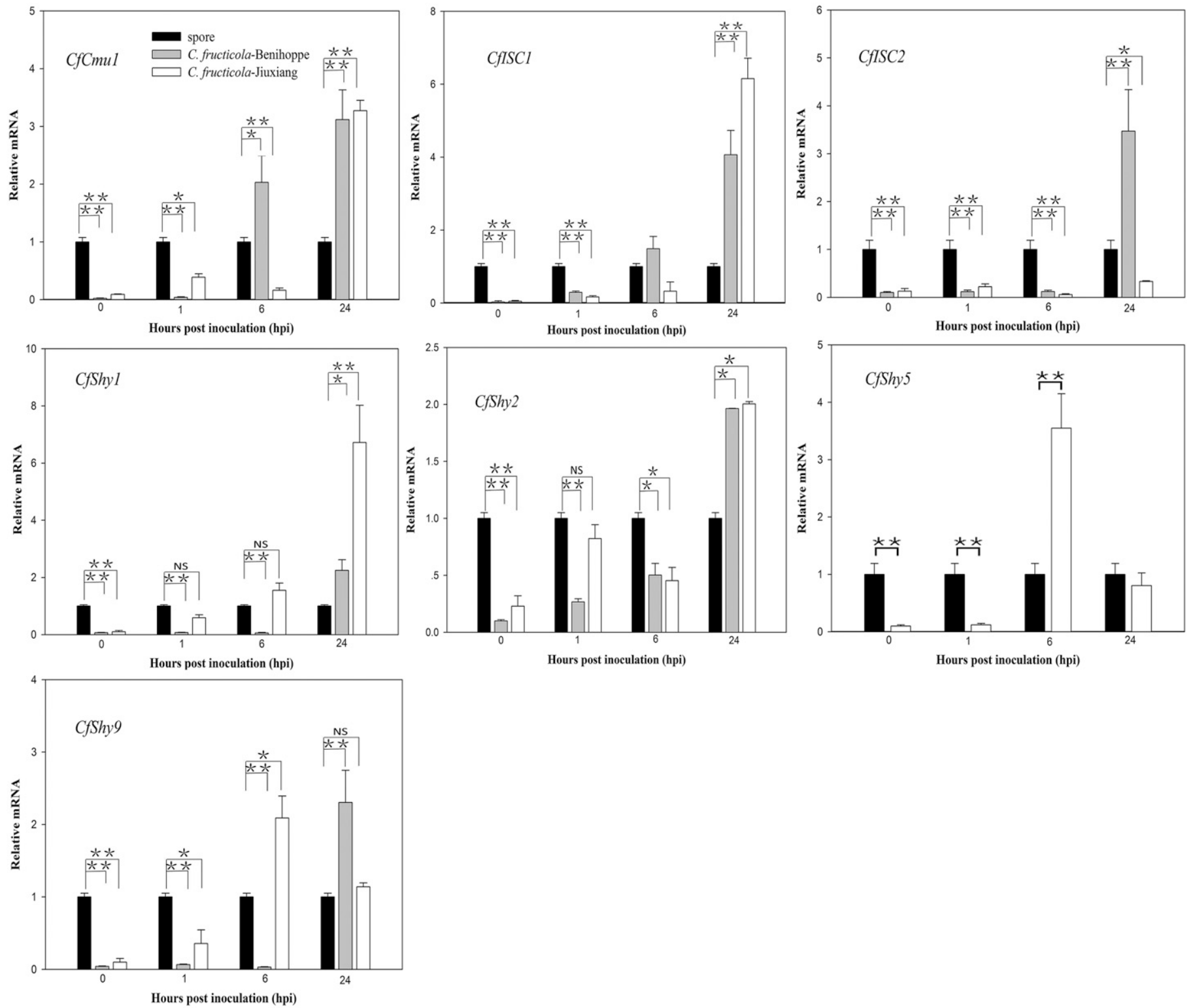

Fig. 4. Transcriptional activation of Colletotrichum fructicola genes targeting host salicylic acid homeostasis during the first $24 \mathrm{~h}$ of infection revealed by quantitative polymerase chain reaction. The expression values of all genes were calculated relative to that in spore (C. fructicola spore; set to one) in each pathosystem. Bars represent mean values of three independent biological experiments \pm standard deviation; $\alpha$-tubulin (CGGC5_11160) and actin (CGGC5_6011) genes were used for normalization. Significant differences between treatments and control are indicated $\left(t\right.$ test). NS, no significant differences. ${ }^{*} P$ value $<0.05$; $* * P$ value $<0.01$. 
loci (CfIsc1, -2, and -7) detected, CfIsc7 was weakly expressed and not up-regulated at $24 \mathrm{hpi}$; thus, it was omitted. The expression patterns of the other seven genes at 1, 6, and 24 hpi in strawberry relative to in axenic culture (conidia for inoculation) are shown in Figure 4. In two varieties, all seven genes were transcriptionally suppressed at $1 \mathrm{hpi}$, but different sets of these genes were inhibited at $6 \mathrm{hpi}$. By $24 \mathrm{hpi}$, the transcription of most of these $C$. fructicola genes was augmented in strawberry. CfCmul (CGGC5_2199) and CfIscl (CGGC5_12510) were significantly activated at $24 \mathrm{hpi}$ in two hosts, but CfIsc2 (CGGC5_7691) expression was only boosted at $24 \mathrm{hpi}$ in Benihoppe. CfShyl (CGGC5_15002) and CfShy2 (CGGC5_ 4272) showed similar patterns in two hosts as CfCmul and CfIsc1; CfShy9 (CGGC5_6647) was similarly expressed as CfIsc2 only in Benihoppe. CfShy5 (CGGC5_9039) showed a special up-regulation limited to Jiuxiang at $6 \mathrm{hpi}$; indeed, mRNA of this gene was not detected in Benihoppe. Considering the contrasting patterns of $C$. fructicola genes at 24 hpi and earlier, we speculate that the decrease in transcript levels from 1 to 6 hpi probably results from strawberry resistance to suppress the pathogen invasion, whereas the activation of these potential effector genes at 24 hpi might indicate that pathogens have successfully broken the host defense systems to a certain extent. Furthermore, CfCmul and CfISCl were provoked earlier in Benihoppe than in Jiuxiang, whereas $C f I S C 2$ was continuously inhibited when infecting Jiuxiang. However, CfShyl, -5, and -9 were expressed earlier or to a higher degree in Jiuxiang than in Benihoppe. These observations suggest that the genes encoding $\mathrm{Cmu}$, Shy, and Isc effectors are all activated in C. fructicola to infect strawberry after $6 \mathrm{hpi}$, although different sets of family members are expressed and display differential dynamic patterns in two hosts.

Subcellular localization analysis suggests that CfShy 1 is a weapon of $C$. fructicola for strawberry invasion. To find out whether the above potential effectors priming strawberry SA homeostasis are activated at the protein level, we first examined the subcellular localization of CfShyl using its GFP fusion protein. CfShyl was chosen, because its transcript level peaked at 24 hpi in both two hosts. We determined the localization pattern of CfShyl during strawberry leaf invasion (at $24 \mathrm{hpi}$ ). The fusion protein of CfShyl (GFP) was observed in the membranes of appressorium and the germ tube (Fig. 5). In addition, the strong punctuate GFP signal of fusion protein was observed in the germ tube. By contrast, the GFP driven by the RP27 promoter (in original pYF11) displayed a strong signal in the cytoplasm of the whole appressoria and germ tube during strawberry invasion. These observations indicate that CfShyl is utilized as a weapon by $C$. fructicola when invading strawberry.

C. fructicola infection triggers the early transcription activity of the hormone-regulated immune signaling network in strawberry. The early transcriptional profiles of a representative set of strawberry genes involved in cytokinin $(\mathrm{CK})$, abscisic acid (ABA), jasmonic acid (JA), and SA signaling pathways were characterized by $\mathrm{qPCR}$ in strawberries after infection. Genes with significant changes in all other pathways except for SA are presented in Figure 6. Genes involved in
DIC

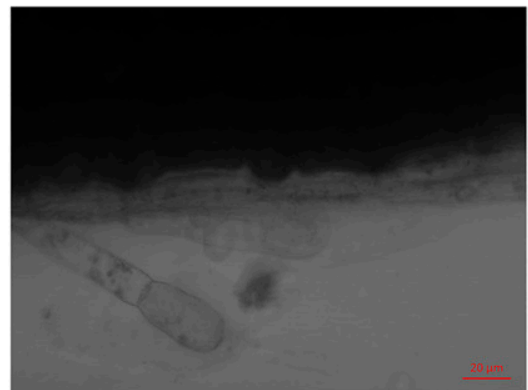

C. fructicola

pYF11
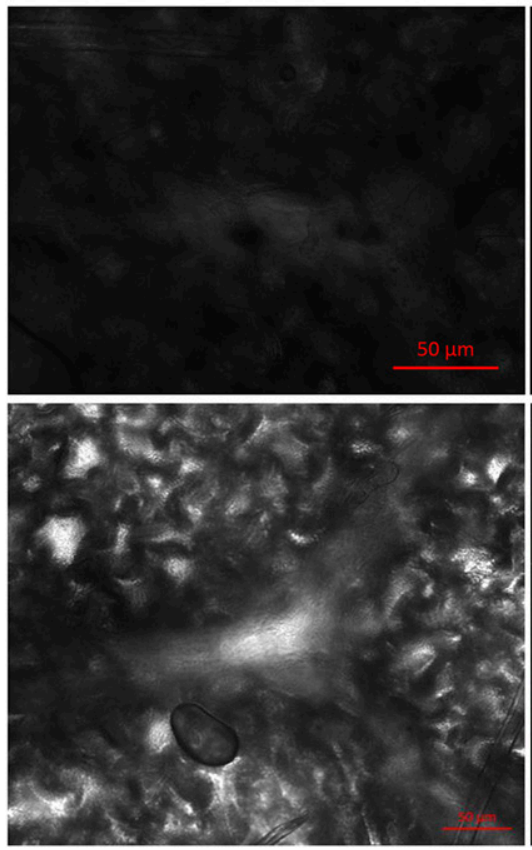

GFP
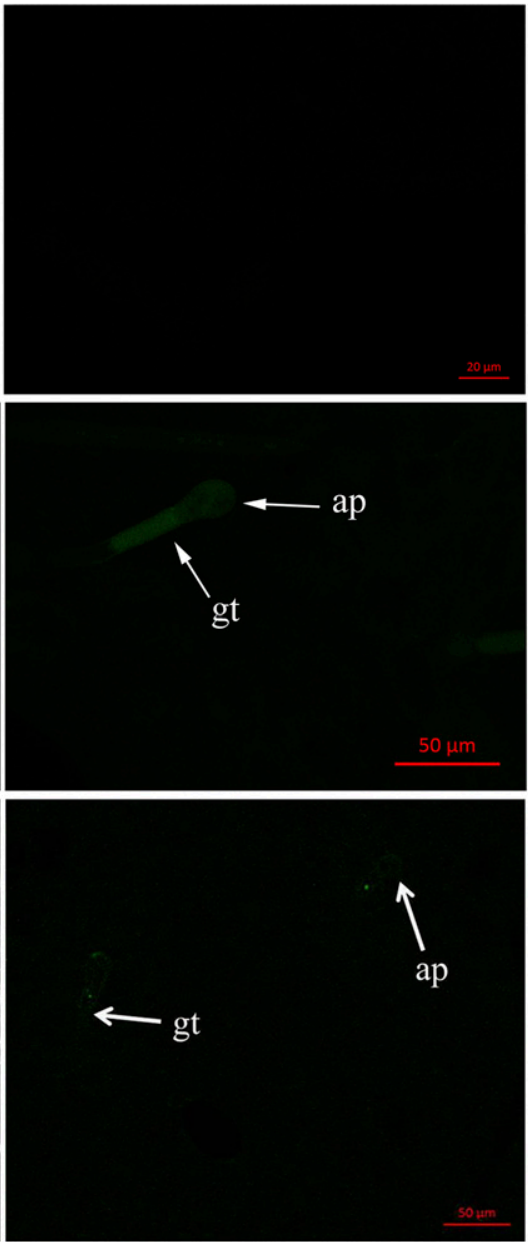

Merged
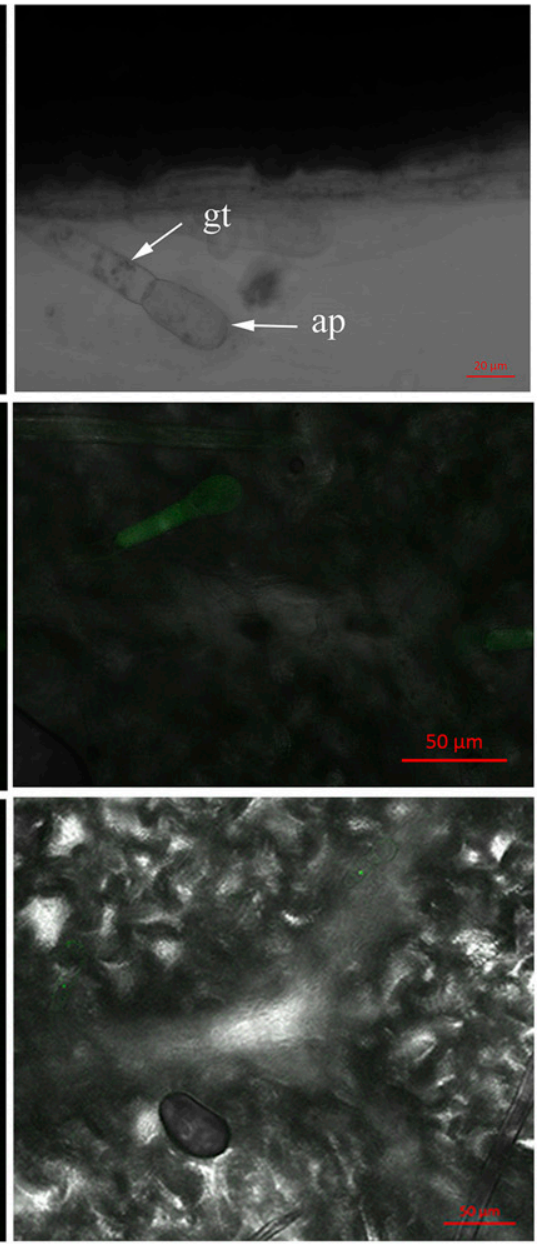

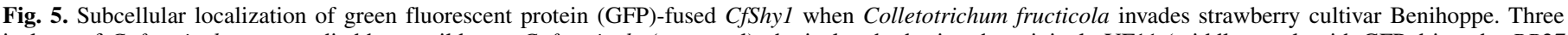

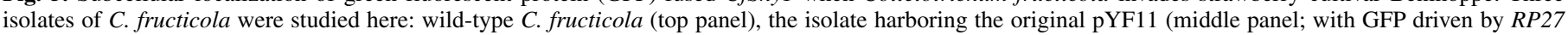

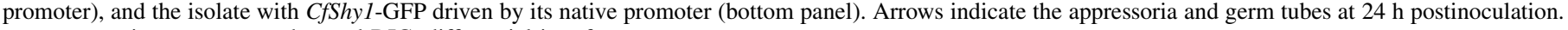
ap, appressorium; gt, germ tube; and DIC, differential interference contrast. 
SA-dependent defense pathway were particularly investigated and are shown in Figure 7.

JA is an important plant hormone regulating plant stress responses. The expression of JASMONATE ZIM DOMAIN 1 (JAZ1), which could bind MYC-related transcriptional activators and inhibit their functions in the JA pathway (Pauwels et al. 2010), was differentially expressed in two varieties (Fig. 6). FaJAZ1 (gene12541) was suppressed at 1 and 6 hpi in Jiuxiang, whereas this gene was soon induced on infection in Benihoppe, indicating that JA-mediated response to $C$. fructicola infection may be multifarious in strawberry. Consistently, JA-responsive gene lipoxygenases (LOX 1; gene05232) displayed activation in Jiuxiang but suppression in Benihoppe at $6 \mathrm{hpi}$. The ABA signaling pathway prominently contributes to plant defense response against both abiotic and biotic stress conditions (Verma et al. 2016). In this work, the ABA-responsive gene FaSalT (gene07088) was similarly induced in two varieties on infection. The up-regulation of an additional ABA-responsive gene FaGlyH (gene14817) was only detected in Jiuxiang at $6 \mathrm{hpi}$. CKs were reported to be involved in defense regulation in 2013 and 2016 (Cao et al. 2016; Jiang et al. 2013). The negative regulators of the CK responses, type A response regulator-like genes FaARR5-1 (gene09501) and FaARR5-2 (gene00726), exhibited similar expression patterns on infection. They were stably up-regulated in Jiuxiang at $24 \mathrm{hpi}$, whereas in Benihoppe, they were suppressed at 1 and 6 hpi, although finally upregulated at $24 \mathrm{hpi}$. The above results suggest that in addition to SA, $\mathrm{JA}, \mathrm{ABA}$, and $\mathrm{CK}$ are coordinately involved in strawberry response to $C$. fructicola, although most genes of these pathways display differential patterns in two varieties.

Several genes in the SA pathway were activated after inoculation. NPR1 is a master regulator of systemic-acquired resistance working as the central transcriptional regulator in the SA pathway (Fu et al. 2012). The potential FaNPRl (gene20070) was differentially transcribed and fluctuated in two varieties (Fig. 7). It was soon induced on infection followed by a quenching at $6 \mathrm{hpi}$, and again, it was up-regulated at $12 \mathrm{hpi}$ in Jiuxiang. The expression of FaNPRl in Benihoppe was only observed at $6 \mathrm{hpi}$ during the first $24 \mathrm{hpi}$. The Senescence-Associated Gene 101 (SAG101) and Pathogen-related (PR) proteins play roles upstream and downstream in the SA signaling pathway, respectively. The up-regulation of strawberry homologous gene FaSAG101a2 (gene28351) was observed in Benihoppe only at $2 \mathrm{hpi}$, whereas in Jiuxiang, it was observed to last from 2 to $12 \mathrm{hpi}$. FaPRI (gene01774), the downstream marker gene of the SA pathway, showed a more stable and higher up-regulation in Benihoppe compared with that in Jiuxiang on infection. Additionally, the expression of $F a P R l$ displayed a clear quenching at 6 hpi in Jiuxiang. Similarly, the transcript levels of an additional pathogenesis-related gene FaPR10 (gene07082) were increased to a higher degree in Benihoppe than in Jiuxiang. The above results suggest that the core defense hormone SA-dependent pathway was not stably activated after $C$. fructicola infection but dynamically regulated with a different rhythm in certain strawberry varieties: induced, quenched, and reactivated at the transcriptional levels accompanied by the changes of genes belonging to other hormonal pathways.
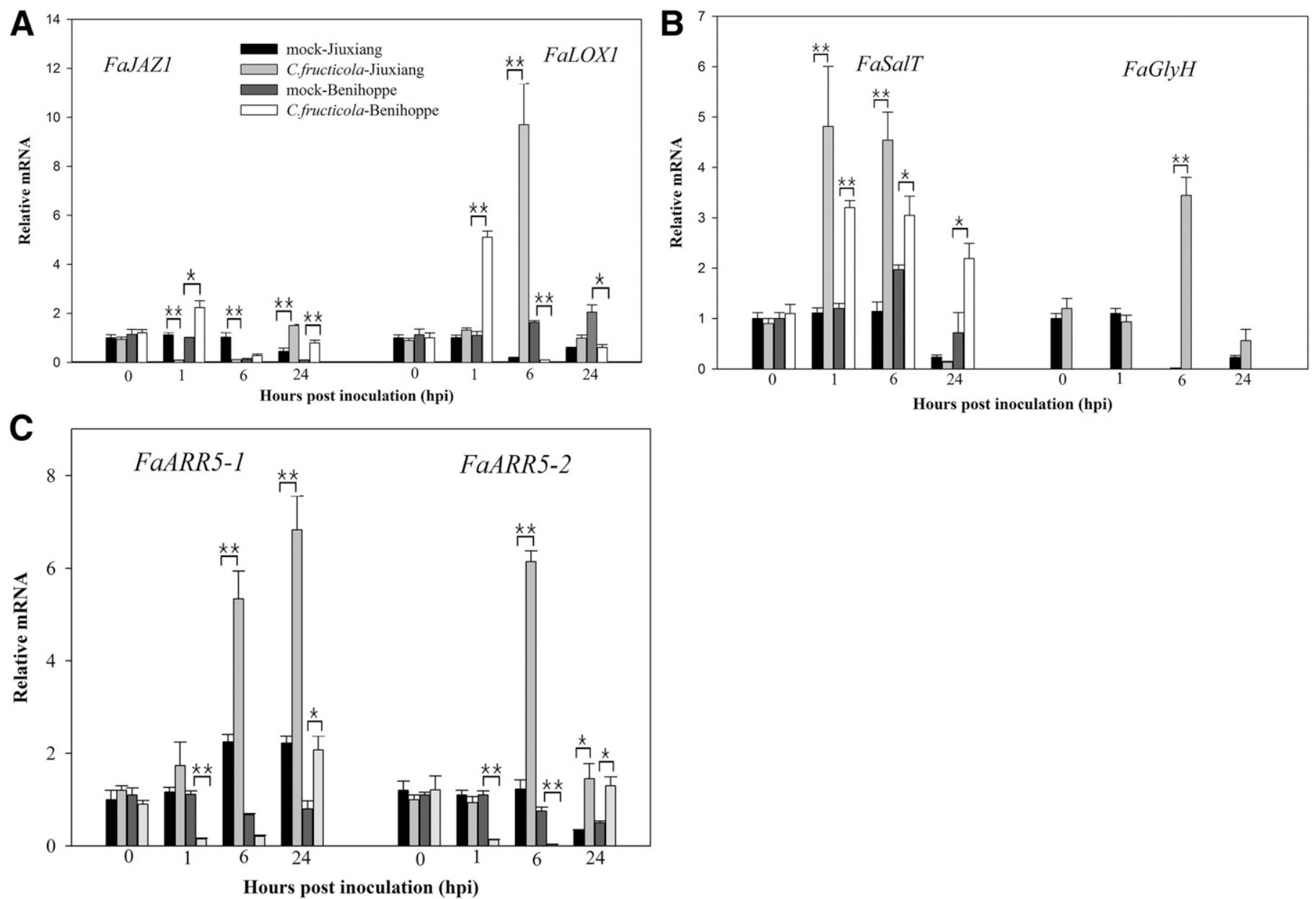

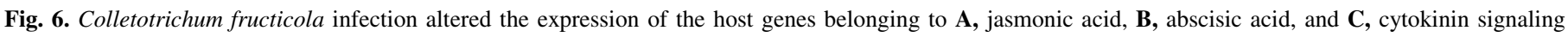

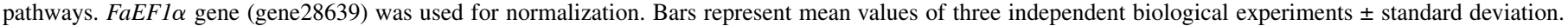
Significant differences between inoculation and mock treatment are indicated $(t$ test $)$. $* P$ value $<0.05 ; * * P$ value $<0.01$. 


\section{DISCUSSION}

The same strain of $C$. fructicola colonizes different strawberry hosts by adopting different invasion strategies. The interaction between pathogen and plant is a mutual process (Vleeshouwers and Oliver 2014). Plants have evolved a variety of immune defense systems. At the same time, pathogens gradually evolve effectors (secreted proteins or toxicity proteins) inhibiting defense, thus increasing plants susceptibility to disease (Cui et al. 2015; Liu et al. 2013; O'Connell et al. 2012). As the core defense regulator against biotrophic and hemibiotrophic pathogens, the host-derived small phenolic compound SA is among the main targets of fungal effectors (Lu et al. 2016). This work suggests that C. fructicola pathogenesis might closely depend on disabling the SA-mediated defense signaling in strawberry. $C$. fructicola could utilize a set of genes encoding effector proteins, including $\mathrm{Cmu}$, Isc, and Shy, to chemically shape and maintain the pathogenic relationship with strawberry.

The strategies that $C$. fructicola used to infect different strawberry varieties are specialized to each host, which can be partially represented by the activation of different sets of genes targeting the host SA defense pathway. For example, the expression of CfISC2 was induced on infecting Benihoppe but not Jiuxiang. Moreover, although $C f C m u l$ expression can be activated in two host varieties, distinct dynamic patterns of this gene were observed during invasion. These observations support the interpretation that,
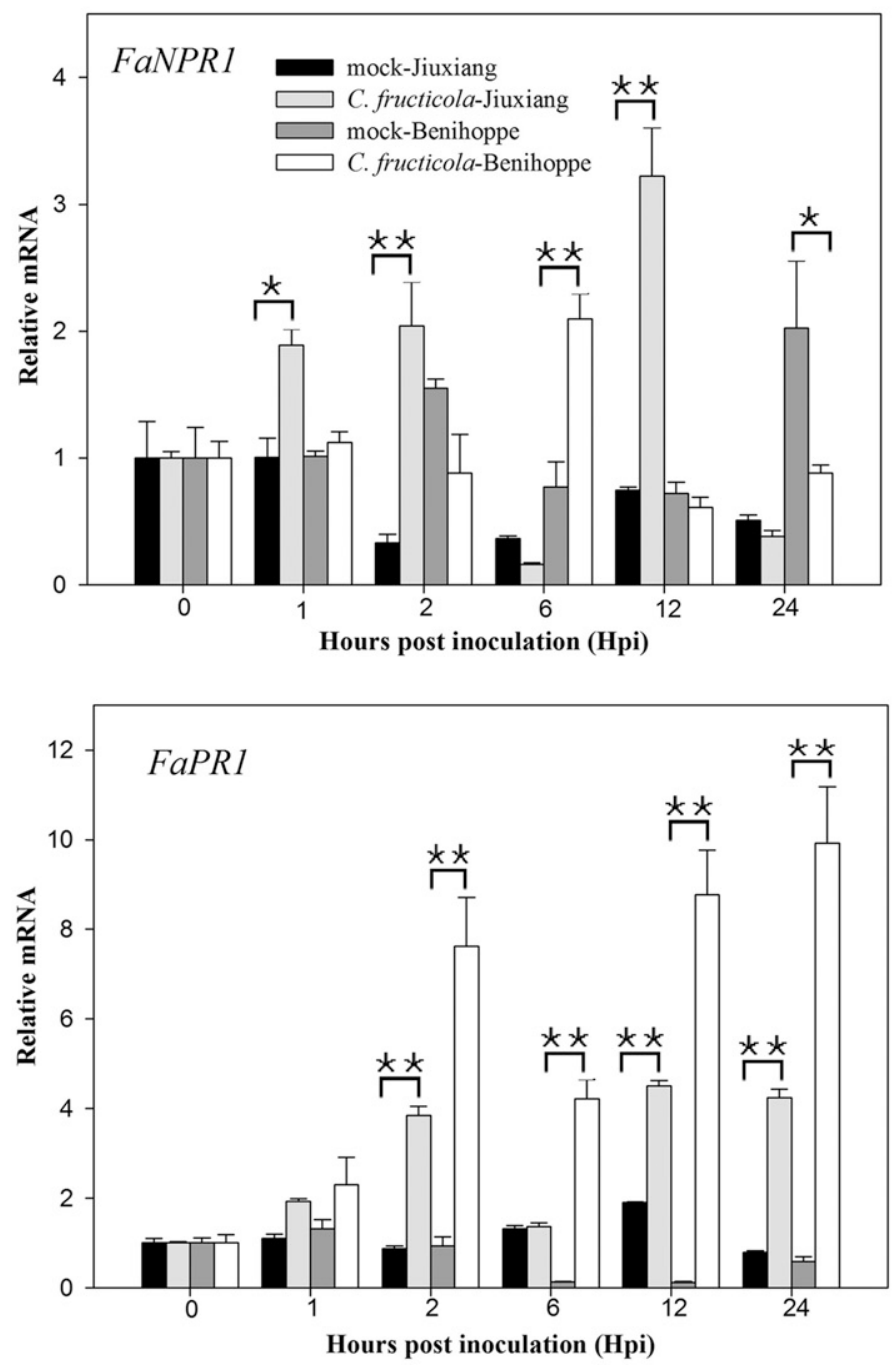

for successful pathogenesis in certain strawberry variety or genotype, C. fructicola could adjust its colonization strategy through an optimized dynamic order of a different set of effector genes.

Strawberry could respond to $C$. fructicola manipulation of SA homeostasis through transient activation of de novo $\mathrm{SA}$ biosynthesis and/or release from integrated $\mathrm{SA}$ metabolites. Several research groups investigated the free SA levels in strawberry during anthracnose pathogenesis. The virulent strain of $C$. acutatum did not induce the temporal accumulation of SA at 48 hpi in cultivars Pájaro and Chandler (Grellet-Bournonville et al. 2012). In the $C$. acutatum-infected crown of cultivar Camarosa, increase of free SA was detected at 3 and 5 dpi (Amil-Ruiz et al. 2016). Consistently, free SA levels were elevated at 3 and 4 dpi in C. fructicola-infected leaves of strawberry cultivar Jiuxiang (Zhang et al. 2018). As for a closer look into the SA changes, an increase of free SA observed in the less susceptible cultivar Sweet Charlie was absent in a more susceptible Jiuxiang at 6 hpi in C. gloeosporioides (Zhang et al. 2016). In this research, a transient increase of free SA levels was revealed in two varieties at $1 \mathrm{hpi}$; this burst of free SA disappeared in Jiuxiang by 2 hpi, whereas it lasted until 6 hpi in Benihoppe.

SA is synthesized from the shikimic acid pathway-derived precursor chorismic acid via two enzymatic routes. The ICS (ICS1/ SA induction-deficient 2)-derived synthesis (mainly occurs in the chloroplast) of SA contributes to $98 \%$ of pathogen-induced SA in
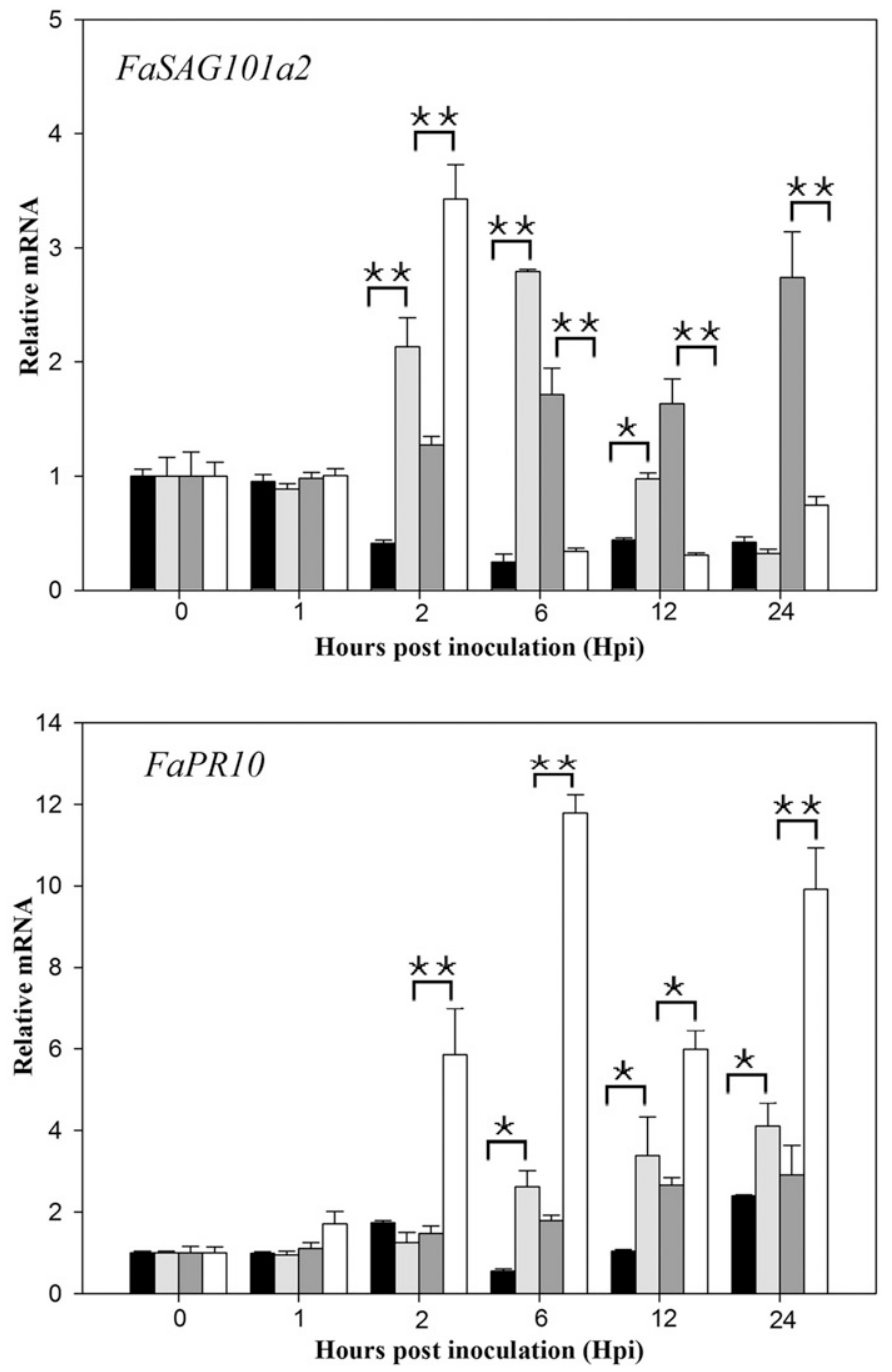

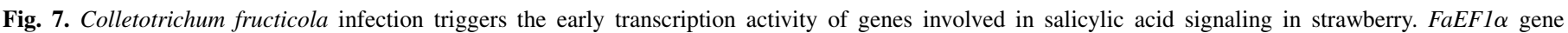

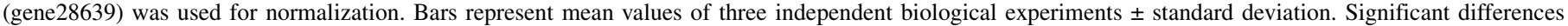
between inoculation and mock treatment are indicated $\left(t\right.$ test). ${ }^{*} P$ value $<0.05 ; * *$ value $<0.01$. 
the genus Arabidopsis (Wildermuth et al. 2001). In 2016, it was reported that both the PAL- and ICS-catalyzed branches contribute equally to the hemibiotrophic pathogen-induced SA accumulation and pathogen defense in soybean (Shine et al. 2016). The transcriptional up-regulation of strawberry homologous genes FaICS1 and FaPAL-1a was observed in both varieties, although with different patterns after $C$. fructicola infection. This activation of putative strawberry SA biosynthesis-related genes suggests that transient de novo biosynthesis of SA from both ICS- and PALcatalyzed branches might be equally involved in strawberry early responses to $C$. fructicola infection.

SA levels in plant can be affected by multiple mechanisms, except for SA biosynthesis. Levels of free SA and conjugated SAG were closely associated during the first $12 \mathrm{hpi}$ in two strawberry varieties, suggesting that the release of SA from SAG might play a certain role in the transient induction of free SA during the first $12 \mathrm{hpi}$ by $C$. fructicola infection. Many proteins have been identified as SABPs, and SA status alters the activity of some SABPs (Klessig et al. 2016). SABP2 encoding MeSA esterase catalyzes the conversion of MeSA back into SA to induce SAR (Tripathi et al. 2010; Vlot et al. 2008). SA binding inhibits the activity of SABP2, resulting in the increase of MeSA (the molecule implicated in SAR) and facilitating its translocation to the uninfected tissue. Transcriptional increase of one SABP2-like gene FaSABP2-3 was detected in Jiuxiang, whereas another homologous gene $F a S A B P 2-2$ was transcriptionally suppressed in two varieties. Thus, the possibility that, in some strawberry variety, the release of SA from MeSA contributes to the early increase of free SA incited by $C$. fructicola infection could not be excluded.

The suppression of $\mathrm{SA}$ levels might be particularly important for $\boldsymbol{C}$. fructicola infection in strawberry. Plant hormones are critical for plant survival under abiotic/biotic stress. The multiple hormones are central players triggering plant immunity, and their signaling pathways are interconnected in a complex network as pathogens try to manipulate host hormone-regulated signaling. It is comprehensible that both SA-dependent and SA-independent pathways are present in the signaling circuitry governing strawberry-induced resistance against $C$. fructicola, similar to the rice/Magnaporthe oryzae interaction (De Vleesschauwer et al. 2008). However, SA could apparently play a central role in these interplays. Based on an HPLC analysis of free SA levels in $>30$ strawberry germplasms in the field, we proposed that the basal SA level is not closely correlated with plant susceptibility to the fungal anthracnose causal agent (unpublished work). This work evidenced that the stable SA level may not count much, but the dynamic SA homeostasis is intricately and delicately controlled in strawberry interplay with C. fructicola. Actually, studies in the genus Arabidopsis indicate that an appropriate SA gradient in the defense zone is critical for SA signaling, because the function of NPR1, the master activator of SAmediated transcriptional reprogramming, requires intermediate SA levels, and also, the SA receptors NPR3 and NPR4 target NPR1 for ubiquitin-mediated protein degradation under high and low SA conditions, respectively (Fu et al. 2012; Saleh et al. 2015; Yan and Dong 2014). In brief, the interplay between SA, NPR1, and NPR3/4 fine tunes NPR1 homeostasis in an SA concentration-dependent manner during pathogen infection.

JA is another important defense-related hormone. LOX and JAZ proteins in JA pathways perceive the bioactive form of jasmonate in the genus Arabidopsis and physically interact with multiple transcription factors to elicit various jasmonate responses (Wasternack and Strnad 2016). The largely antagonistic functions of JA and SA have been suggested for plant response to pathogen infection (Verma et al. 2016). There also exists cooperative interplay between JA and SA during early ETI. In parallel to SA accumulation in the genus Arabidopsis, the endogenous JA level also increases during ETI induction, which may reduce plant susceptibility to necrotrophic pathogens in the neighboring tissue; this elevation of JA is dependent on the degradation of JAZ mediated by direct interaction with NPR3-NPR4 (Liu et al. 2016).

A recent study of rice plasma membrane proteomics revealed a sequential activation of different hormone-mediated responses to blast fungi $M$. oryzae: at $24 \mathrm{hpi}$, the activation of ABA signaling compromised disease resistance, and at $48 \mathrm{hpi}$, CK signaling upregulated the sugar transporter genes (e.g., OsSWEET11) expression and nutrient efflux for massive growth of fungi in rice apoplasm (Cao et al. 2016). ABA enhancement of the susceptibility to M. grisea is through suppressing OsWRKY45 and OsNPR1 of the SA signaling pathway (Jiang et al. 2010). Although CK treatment alone induces neither SA accumulation nor disease resistance, CK
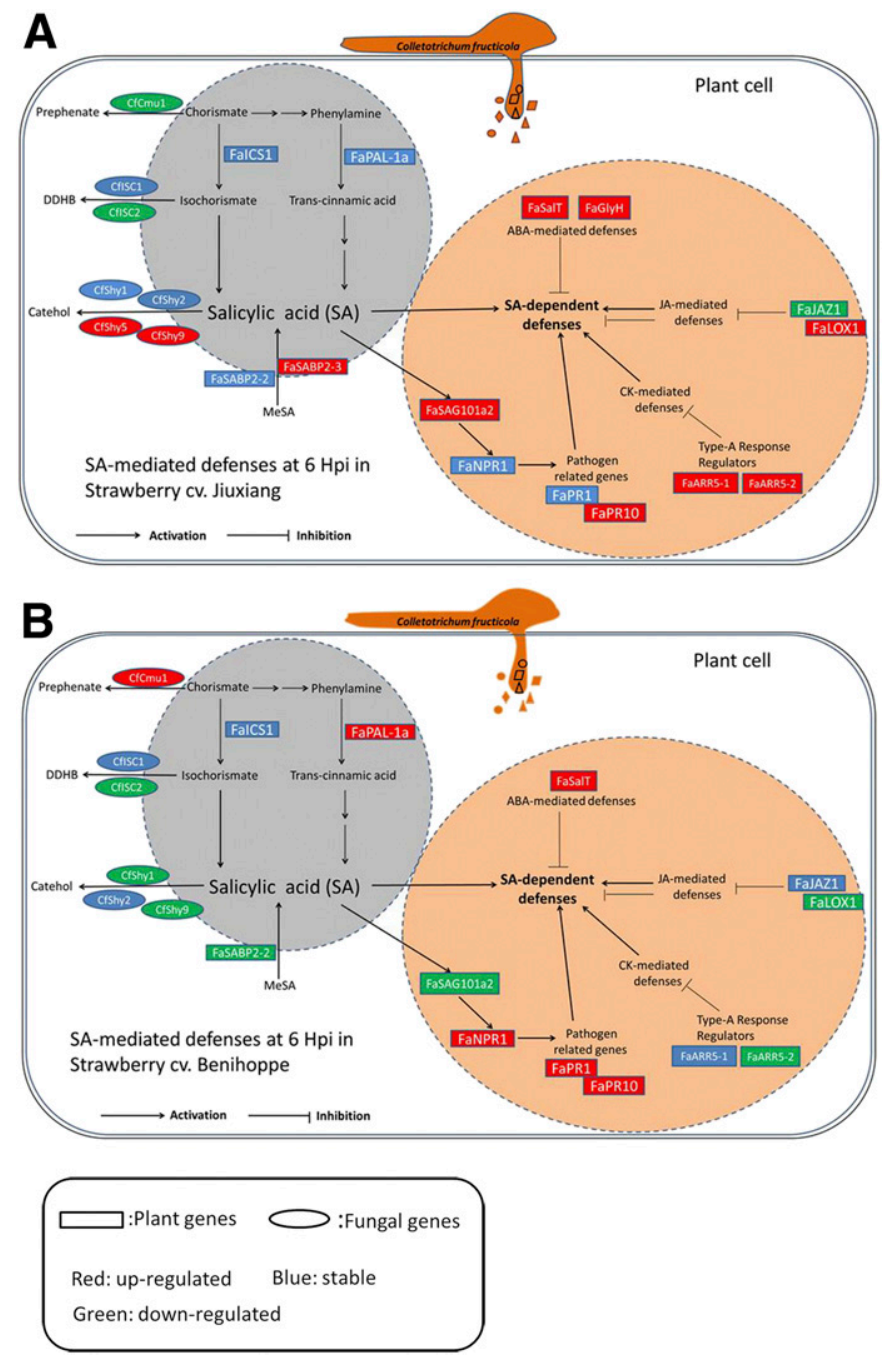

Fig. 8. Schematic illustration of the transcriptional events involved in salicylic acid (SA)-mediated defenses at $6 \mathrm{~h}$ postinoculation (hpi) of Colletotrichum fructicola. A, In cultivar Jiuxiang. B, In cultivar Benihoppe. In strawberry, SA could be produced via the isochorismate synthase (ICS) pathway in plastids and through the phenylalanine ammonia lyase (PAL) pathway in the cytosol. Secreted chorismate mutase 1-2 from $C$. fructicola can be taken up by strawberry cells, and it rechannels chorismic acid from plastids to the cytosol, thus lowering SA levels. Also, fungus secrets isochorismatases Isc1-10, converting isochorismate into 2,3-dihydro-2,3-dihydroxybenzoate (DDHB) and pyruvate. Additionally, $C$. fructicola harbors a family with 13 gene members encoding Salicylate hydroxylase Shy1-13, which can degrade SA. Fungal proteins are indicated by gray ovals, and plant proteins are indicated by black squares. C. fructicola infection can modify the SA-mediated defenses via activation of the antagonistic or synergistic jasmonic acid (JA) and abscisic acid (ABA) signaling pathways. The plant hormone cytokinin (CK) could contribute positively to SA-dependent defenses. Arrows indicate positive effects; inhibition lines represent negative effects. Ovals indicate fungal genes, and squares indicate strawberry genes. Red characters indicate up-regulation, and green characters indicate down-regulation. SABP2, salicylic acid-binding protein 2 . 
acts synergistically with SA to activate defense gene expression (Jiang et al. 2013).

Based on this work and previous studies on hormonal interplay between plant and hemibiotrophic pathogens, we propose a schematic representation of early molecular components involved in the SA signaling pathway of strawberry/C. fructicola interaction (Fig. 8). A glimpse of the transcriptional events focused on SAmediated defenses at $6 \mathrm{hpi}$ in certain strawberry variety sheds some light on the importance of the delicate SA homeostasis and host specialized interplay with the same isolate of anthracnose pathogen C. fructicola.

Conclusions. For the first time, the early delicate SA homeostasis and the transcriptional profiles of SA-relevant strawberry defense genes as well as anthracnose pathogen effector genes are presented. A close view on strawberry/C. fructicola interaction in the first day will assist future studies in increasing the understanding of strawberry security flaws and $C$. fructicola virulence. The very early incitation of SA accumulation followed by a fast quenching is commonly identified in different strawberry genotypes on $C$. fructicola infection. At the transcriptional level, transient de novo SA biosynthesis from both ICS- and PALmediated pathways and the release from SA-related metabolites are found to work together for the intricate control of SA homeostasis in strawberry. Activation of seven effector genes encoding three types of effectors ( $\mathrm{Cmu}$, Shy, and Isc) targeting host SA homeostasis is reported in strawberry/C. fructicola interplay. We hope that our work presented here provides some novel clues for future exploration of strawberry resistance to its anthracnose fungal pathogen.

\section{ACKNOWLEDGMENTS}

We thank H. Yoo of Duke University for the kind advice on SA extraction and measurement in strawberry materials, H. Zhang of Nanjing Agricultural University for valuable discussion and the gift of pYF11, and our colleague J. Wan for confocal observation.

\section{LITERATURE CITED}

Amil-Ruiz, F., Blanco-Portales, R., Muñoz-Blanco, J., and Caballero, J. L. 2011. The strawberry plant defense mechanism: A molecular review. Plant Cell Physiol. 52:1873-1903.

Amil-Ruiz, F., Garrido-Gala, J., and Blanco-Portales, R. 2013. Identification and validation of reference genes for transcript normalization in strawberry (Fragaria $\times$ ananassa) defense responses. PLoS One 8:e70603.

Amil-Ruiz, F., Garrido-Gala, J., Gadea, J., Blanco-Portales, R., Muñoz-Mérida, A., Trelles, O., de Los Santos, B., Arroyo, F. T., Aguado-Puig, A., Romero, F., Mercado, J. Á., Pliego-Alfaro, F., Muñoz-Blanco, J., Caballero, J. L. 2016. Partial activation of SA-and JA-defensive pathways in strawberry upon Colletotrichum acutatum interaction. Front. Plant Sci. 7: 1036.

Asai, S., and Shirasu, K. 2015. Plant cells under siege: Plant immune system versus pathogen effectors. Curr. Opin. Plant Biol. 28:1-8.

Baroncelli, R., Zapparata, A., Sarrocco, S., Sukno, S. A., Lane, C. R., Thon, M. R., Vannacci, G., Holub, E., and Sreenivasaprasad, S. 2015. Molecular diversity of anthracnose pathogen populations associated with UK strawberry production suggests multiple introductions of three different Colletotrichum species. PLoS One 10:e0129140.

Buddie, A. G., Martinez-Culebras, P., Bridge, P. D., García, M. D., Querol, A., Cannon, P. F., and Monte, E. 1999. Molecular characterization of Colletotrichum strains derived from strawberry. Mycol. Res. 103: 385-394.

Cao, J., Yang, C., Li, L., Jiang, L., Wu, Y., Wu, C., Bu, Q., Xia, G., Liu, X., Luo, Y., and Liu, J. 2016. Rice plasma membrane proteomics reveals Magnaporthe oryzae promotes susceptibility by sequential activation of host hormone signaling pathways. Mol. Plant-Microbe Interact. 29: 902-913.

Cui, H., Tsuda, K., and Parker, J. E. 2015. Effector-triggered immunity: From pathogen perception to robust defense. Annu. Rev. Plant Biol. 66: 487-511.

De Vleesschauwer, D., Djavaheri, M., Bakker, P. A., and Höfte, M. 2008. Pseudomonas fluorescens WCS374r-induced systemic resistance in rice against Magnaporthe oryzae is based on pseudobactin-mediated priming for a salicylic acid-repressible multifaceted defense response. Plant Physiol. 148:1996-2012.

Denoyes-Rothan, B., Guerin, G., Delye, C., Smith, B., Minz, D., Maymon, M., and Freeman, S. 2003. Genetic diversity and pathogenic variability among isolates of Colletotrichum species from strawberry. Phytopathology 93: 219-228.

Djamei, A., Schipper, K., Rabe, F., Ghosh, A., Vincon, V., and Kahnt, J. 2011. Metabolic priming by a secreted fungal effector. Nature 478:395-398.

Freeman, S., and Katan, T. 1997. Identification of Colletotrichum species responsible for anthracnose and root necrosis of strawberry in Israel. Phytopathology 87:516-521.

Fu, Z. Q., Yan, S., Saleh, A., Wang, W., Ruble, J., and Oka, N. 2012. NPR3 and NPR4 are receptors for the immune signal salicylic acid in plants. Nature 486:228-232.

Gan, P., Ikeda, K., Irieda, H., Narusaka, M., O’Connell, R. J., Narusaka, Y., Takano, Y., Kubo, Y., and Shirasu, K. 2013. Comparative genomic and transcriptomic analyses reveal the hemibiotrophic stage shift of Colletotrichum fungi. New Phytol. 197:1236-1249.

Garrido, C., Carbu, M., Fernandez-Acero, F. J., Budge, G., Vallejo, I., Colyer, A., and Cantoral, J. M. 2008. Isolation and pathogenicity of Colletotrichum spp. causing anthracnose of strawberry in south west Spain. Eur. J. Plant Pathol. 120:409-415.

Gimenez-Ibanez, S., and Solano, R. 2013. Nuclear jasmonate and salicylate signaling and crosstalk in defense against pathogens. Front. Plant Sci. 4:72.

Grellet-Bournonville, C. F., Martinez-Zamora, M. G., Castagnaro, A. P., and Díaz-Ricci, J. C. 2012. Temporal accumulation of salicylic acid activates the defense response against Colletotrichum in strawberry. Plant Physiol. Biochem. 54:10-16.

Han, Y. C., Zeng, X. G., Xiang, F. Y., Ren, L., Chen, F. Y., and Gu, Y. C. 2016. Distribution and characteristics of Colletotrichum spp. associated with anthracnose of strawberry in Hubei, China. Plant Dis. 100:996-1006.

Huang, L., Zhang, S., Yin, Z., Liu, M., Li, B., Zhang, H., Zheng, X., Wang, P., and Zhang, Z. 2017. Movrp1, a putative verprolin protein, is required for asexual development and infection in the rice blast fungus Magnaporthe oryzae. Sci. Rep. 7:41148.

Hyde, K. D., Cai, L., Cannon, P. F., Crouch, J. A., Crous, P. W., Damm, U., Goodwin, P. H., Chen, H., Johnston, P. R., Jones, E. B. G., Liu, Z. Y., McKenzie, E. H. C., Moriwaki, J., Noireung, P., Pennycook, S. R., Pfenning, L. H., Prihastuti, H., Sato, T., Shivas, R. G., Tan, Y. P., Taylor, P. W. J., Weir, B. S., Yang, Y. L., and Zhang, J. Z. 2009. Colletotrichum-names in current use. Fungal Divers. 39:147-182.

Jelev, Z. J., Bobev, S. G., Minz, D., Maymon, M., and Freeman, S. 2008. Characterization of Colletotrichum species causing strawberry anthracnose in Bulgaria. J. Phytopathol. 156:668-677.

Jiang, C. J., Shimono, M., Sugano, S., Kojima, M., Liu, X., Inoue, H., Sakakibara, H., and Takatsuji, H. 2013. Cytokinins act synergistically with salicylic acid to activate defense gene expression in rice. Mol. PlantMicrobe Interact. 26:287-296.

Jiang, C. J., Shimono, M., Sugano, S., Kojima, M., Yazawa, K., and Yoshida, R. 2010. Abscisic acid interacts antagonistically with salicylic acid signaling pathway in rice-Magnaporthe grisea interaction. Mol. PlantMicrobe Interact. 23:791-798.

Klessig, D. F., Tian, M., and Choi, H. W. 2016. Multiple targets of salicylic acid and its derivatives in plants and animals. Front. Immunol. 7:1.

Li, J., Zhang, Q. Y., Gao, Z. H., Wang, F., Duan, K., Ye, Z. W., and Gao, Q. H. 2013. Genome-wide identification and comparative expression analysis of NBS-LRR-encoding genes upon Colletotrichum gloeosporioides infection in two ecotypes of Fragaria vesca. Gene 527:215-227.

Liu, L., Sonbol, F. M., Huot, B., Gu, Y., Withers, J., Mwimba, M., Yao, J., He, S. Y., and Dong, X. 2016. Salicylic acid receptors activate jasmonic acid signalling through a non-canonical pathway to promote effector-triggered immunity. Nat. Commun. 7:13099.

Liu, T., Song, T., Zhang, X., Yuan, H., Su, L., Li, W., Xu, J., Liu, S., Chen, L., Chen, T., Zhang, M., Gu, L., Zhang, B., Dou, D. 2014. Unconventionally secreted effectors of two filamentous pathogens target plant salicylate biosynthesis. Nat. Commun. 5:4686.

Liu, W., Liu, J., Ning, Y., Ding, B., Wang, X., Wang, Z., and Wang, G. L. 2013. Recent progress in understanding PAMP-and effector-triggered immunity against the rice blast fungus Magnaporthe oryzae. Mol. Plant 6:605-620.

Lu, H., Greenberg, J. T., and Holuigue, L. 2016. Editorial: Salicylic acid signaling networks. Front. Plant Sci. 7:238.

O'Connell, R. J., Thon, M. R., Hacquard, S., Amyotte, S. G., Kleemann, J., and Torres, M. F. 2012. Lifestyle transitions in plant pathogenic Colletotrichum fungi deciphered by genome and transcriptome analyses. Nat. Genet. 44:1060-1065.

Patkar, R. N., and Naqvi, N. I. 2017. Fungal manipulation of hormoneregulated plant defense. PLoS Pathog 13:e1006334.

Pauwels, L., Barbero, G. F., Geerinck, J., Tilleman, S., Grunewald, W., and Pérez, A. C. 2010. NINJA connects the co-repressor TOPLESS to jasmonate signalling. Nature 464:788-791. 
Pieterse, C. M., Van der Does, D., Zamioudis, C., Leon-Reyes, A., and Van Wees, S. C. 2012. Hormonal modulation of plant immunity. Annu. Rev. Cell. Dev. Bi. 28:489-521.

Rabe, F., Ajami-Rashidi, Z., Doehlemann, G., Kahmann, R., and Djamei, A. 2013. Degradation of the plant defence hormone salicylic acid by the biotrophic fungus Ustilago maydis. Mol. Microbiol. 89:179-188.

Rahman, M., Ojiambo, P., and Louws, F. 2015. Initial inoculum and spatial dispersal of Colletotrichum gloeosporioides, the causal agent of strawberry anthracnose crown rot. Plant Dis. 99:80-86.

Ren, X. J., Liang, Y., Lu, J. P., Yang, B. R., Xu, J. Y., and Dai, F. M. 2008. Identification of Colletotrichum species from strawberry in Shanghai. Acta Phytopathol. Sin. 38:325-328.

Saleh, A., Withers, J., Mohan, R., Marqués, J., Gu, Y., and Yan, S. 2015. Posttranslational modifications of the master transcriptional regulator NPR1 enable dynamic but tight control of plant immune responses. Cell Host Microbe 18:169-182.

Shine, M. B., Yang, J. W., El-Habbak, M., Nagyabhyru, P., Fu, D. Q., and Navarre, D. 2016. Cooperative functioning between phenylalanine ammonia lyase and isochorismate synthase activities contributes to salicylic acid biosynthesis in soybean. New Phytol. 212:627-636.

Silva, K. J. P., Brunings, A., Peres, N. A., Mou, Z., and Folta, K. M. 2015. The Arabidopsis NPR1 gene confers broad-spectrum disease resistance in strawberry. Transgenic Res. 24:693-704.

Tanaka, S., Han, X., and Kahmann, R. 2015. Microbial effectors target multiple steps in the salicylic acid production and signaling pathway. Front. Plant Sci. 6:349.

Tripathi, D., Jiang, Y. L., and Kumar, D. 2010. SABP2, a methyl salicylate esterase is require for the systemic acquired resistance induced by acibenzolar-S-methyl in plants. FEBS Lett. 584:3458-3463.

Verma, V., Ravindran, P., and Kumar, P. P. 2016. Plant hormone-mediated regulation of stress responses. BMC Plant Biol. 16:86.

Vleeshouwers, V. G., and Oliver, R. P. 2014. Effectors as tools in disease resistance breeding against biotrophic, hemibiotrophic, and necrotrophic plant pathogens. Mol. Plant-Microbe Interact. 27:196-206.
Vlot, A. C., Liu, P. P., Cameron, R. K., Park, S. W., Yang, Y., and Kumar, D. 2008. Identification of likely orthologs of tobacco salicylic acid-binding protein 2 and their role in systemic acquired resistance in Arabidopsis thaliana. Plant J. 56:445-456.

Vos, I. A., Pieterse, C. M. J., and Wees, S. C. M. 2013. Costs and benefits of hormone-regulated plant defences. Plant Pathol. 62:43-55.

Wasternack, C., and Strnad, M. 2016. Jasmonate signaling in plant stress responses and development-active and inactive compounds. N. Biotechnol. 33:604-613.

Weir, B. S., Johnston, P. R., and Damm, U. 2012. The Colletotrichum gloeosporioides species complex. Stud. Mycol. 73:115-180.

Widiastuti, A., Yoshino, M., Saito, H., Maejima, K., Zhou, S., Odani, H., Narisawa, K., Hasegawa, M., Nitta, Y., and Sato, T. 2013. Heat shockinduced resistance in strawberry against crown rot fungus Colletotrichum gloeosporioides. Physiol. Mol. Plant Pathol. 84:86-91.

Wildermuth, M. C., Dewdney, J., and Wu, G. 2001. Isochorismate synthase is required to synthesize salicylic acid for plant defence. Nature 414:562-565.

Xie, L., Zhang, J. Z., and Wan, Y. 2010. Identification of Colletotrichum spp. isolated from strawberry in Zhejiang Province and Shanghai City, China. J. Zhejiang Univ. Sci. B 11:61-70.

Yan, S., and Dong, X. 2014. Perception of the plant immune signal salicylic acid. Curr. Opin. Plant Biol. 20:64-68.

Zhang, L., Huang, X., He, C., Zhang, Q. Y., Zou, X., Duan, K., and Gao, Q. 2018. Novel fungal pathogenicity and leaf defense strategies are revealed by simultaneous transcriptome analysis of Colletotrichum fructicola and strawberry infected by this fungus. Front. Plant Sci. 9:434.

Zhang, Q. Y., Zhang, L. Q., Song, L. L., Duan, K., Li, N., Wang, Y. X., and Gao, Q. H. 2016. The different interactions of Colletotrichum gloeosporioides with two strawberry varieties and the involvement of salicylic acid. Hortic. Res. 3:16007.

Zheng, X. Y., Zhou, M., Yoo, H., Pruneda-Paz, J. L., Spivey, N. W., Kay, S. A., and Dong, X. 2015. Spatial and temporal regulation of biosynthesis of the plant immune signal salicylic acid. Proc. Natl. Acad. Sci. U.S.A. 112: 9166-9173. 